IJMMS 32:8 (2002) 471-479

PII. S0161171202203361

http://ijmms.hindawi.com

(c) Hindawi Publishing Corp.

\title{
RATE OF CONVERGENCE ON BASKAKOV-BETA-BEZIER OPERATORS FOR BOUNDED VARIATION FUNCTIONS
}

\author{
VIJAY GUPTA
}

Received 28 March 2002

\begin{abstract}
We introduce a new sequence of linear positive operators $B_{n, \alpha}(f, x)$, which is the Bezier variant of the well-known Baskakov Beta operators and estimate the rate of convergence of $B_{n, \alpha}(f, x)$ for functions of bounded variation. We also propose an open problem for the readers.
\end{abstract}

2000 Mathematics Subject Classification: 41A17, 41A25.

1. Introduction. Let $H[0, \infty)=\{f: f$ is locally bounded on $(0, \infty)$ and $|f(t)| \leq$ $\left.M(1+t)^{\beta}, M>0, \beta \in \mathbb{N}_{0}\right\}$, then, for $f \in H[0, \infty)$, Baskakov-Durrmeyer operators are defined as

$$
V_{n}(f, x)=(n-1) \sum_{k=0}^{\infty} p_{n, k}(x) \int_{0}^{\infty} p_{n, k}(x) f(t) d t, \quad n \in \mathbb{N}, x \in[0, \infty),
$$

where

$$
p_{n, k}(t)=\left(\begin{array}{c}
n+k-1 \\
k
\end{array}\right) \frac{x^{k}}{(1+x)^{n+k}} .
$$

The operators result from the classical Baskakov operators

$$
\hat{V}_{n}(f, x)=\sum_{k=0}^{\infty} p_{n, k}(x) f\left(\frac{k}{n}\right)
$$

by replacing the discrete value $f(k / n)$ by the integral $(n-1) \int_{0}^{\infty} p_{n, k}(t) f(t) d t$ in order to approximate Lebesgue integrable functions on the interval $[0, \infty)$. Some approximation properties of the operators (1.1) were discussed in $[6,7,8]$.

In [2, 3], the author defined another modification of the Baskakov operators with the weight functions of Beta operators so as to approximate Lebesgue integrable functions on $[0, \infty)$. For $f \in H[0, \infty)$, Baskakov Beta operators are defined as

$$
B_{n}(f, x)=\sum_{k=0}^{\infty} p_{n, k}(x) \int_{0}^{\infty} b_{n, k}(t) f(t) d t, \quad n \in \mathbb{N}, x \in[0, \infty),
$$

where $p_{n, k}(x)$ is as defined in (1.1), $b_{n, k}(t)=t^{k} /\left(B(k+1, n)(1+t)^{n+k+1}\right)$, and $B(k+$ $1, n)=k !(n-1) ! /(n+k) !$

It was observed in [2] that the integral modification of the Baskakov operators defined by (1.4) gives better results than the operators (1.1), and some approximation properties for the operators $B_{n}$ become simpler in comparison to the operators $V_{n}$, 
for example, in the estimation of the rate of convergence of bounded variation functions we need not to use result of the type [8, Lemma 5] for the operators (1.4). This motivated further study of the operators $B_{n}$. For $f \in H[0, \infty), \alpha \geq 1$, we introduce the Bezier variant of the operators (1.4) as follows:

$$
B_{n, \alpha}(f, x)=\sum_{k=0}^{\infty} Q_{n, k}^{(\alpha)}(x) \int_{0}^{\infty} b_{n, k}(t) f(t) d t, \quad x \in[0, \infty),
$$

where $Q_{n, k}^{(\alpha)}(x)=J_{n, k}^{\alpha}(x)-J_{n, k+1}^{\alpha}(x)$ and $\sum_{j=k}^{\infty} p_{n, j}(x)=J_{n, k}(x)$ is the Baskakov basis function. Obviously, $B_{n, \alpha}(1, x)=1$, and, particularly when $\alpha=1$, the operators (1.4) reduce to the operators (1.4). It is observed that $B_{n, \alpha}(f, x)$ is the sequence of linear positive operators. Some basic properties of $J_{n, k}(x)$ are as follows:

(i) $J_{n, k}(x)-J_{n, k+1}(x)=p_{n, k}(x), k=0,1,2,3, \ldots$,

(ii) $J_{n, k}^{\prime}(x)=n p_{n+1, k-1}(x), k=1,2,3, \ldots$,

(iii) $J_{n, k}(x)=n \int_{0}^{x} p_{n+1, k-1}(t) d t, k=0,1,2,3, \ldots$,

(iv) $\sum_{k=1}^{\infty} J_{n, k}(x)=n \int_{0}^{x} \sum_{k=1}^{\infty} p_{n+1, k-1}(t) d t=n x$,

(v) $J_{n, 0}(x)>J_{n, 1}(x)>J_{n, 2}(x)>\cdots>J_{n, k}(x)>J_{n, k+1}(x)>\cdots$,

for every natural number $k, 0<J_{n, k}(x)<1$ and $J_{n, k}(x)$ increases strictly on $[0, \infty)$. These properties can be obtained easily by direct computation.

Bojanić and Vulleumier [1] estimated the rate of convergence of Fourier series of functions of bounded variation. Recently, Zeng and Chen [11] estimated the rate of convergence of the Durrmeyer-Bezier operators for functions of bounded variation. Zeng and Gupta [12] and Zeng [10] estimated the rate of approximation for the Bezier variant of classical Baskakov and Szász operators, respectively, at those points at which one-sided limits $f(x \pm)$ exist. In the present paper, we estimate the rate of convergence of the operators $B_{n, \alpha}(f, x)$ for functions of bounded variation. The approximation properties for the operators $B_{n, \alpha}(f, x)$ are different.

THEOREM 1.1. Let $f \in H[0, \infty)$, and let at a fixed point $x \in(0, \infty)$, the one-sided limits $f(x \pm)$, exist. Then, for $\alpha \geq 1, \lambda>2, x \in(0, \infty)$ and for $n>\max \{1+\beta, \mathbb{N}(\lambda, x)\}$, we have

$$
\begin{aligned}
\left|B_{n, \alpha}(f, x)-\left[\frac{1}{\alpha+1} f(x+)+\frac{\alpha}{\alpha+1} f(x-)\right]\right| \\
\leq|f(x+)-f(x-)| \cdot \frac{\alpha \sqrt{1+x}}{\sqrt{2 e n x}}+\frac{\alpha[3 \lambda+(1+3 \lambda) x]}{n x} \\
\quad \times \sum_{k=1}^{n} V_{x-x / \sqrt{k}}^{x+x / \sqrt{k}}\left(g_{x}\right)+M \alpha\left(2^{\beta}-1\right) \frac{(1+x)^{\beta}}{x^{2 \beta}} O\left(n^{-\beta}\right)+\frac{2 M \alpha \lambda(1+x)^{\beta+1}}{n x},
\end{aligned}
$$

where

$$
g_{x}(t)= \begin{cases}f(t)-f(x-), & 0 \leq t<x \\ 0, & t=x, \\ f(t)-f(x+), & x<t<\infty\end{cases}
$$

and $V_{a}^{b}\left(g_{x}\right)$ is the total variation of $g_{x}$ on $[a, b]$. 
2. Auxiliary results. In this section, we give certain results, which are necessary to prove the main result.

Yuankwei and Shunsheng [8] gave the following inequality for Baskakov basis functions. For $x \in(0, \infty)$ and $k \in \mathbb{N}$, there holds

$$
p_{n, k}(t) \leq \frac{33}{\sqrt{n}}\left(\frac{1+x}{x}\right)^{3 / 2}
$$

The above bound discussed in [8] was not sharp bound. Recently, Zeng [9] estimated the exact bounds for Bernstein basis functions and Meyer-Konig-Zeller basis functions. Using the inequality estimate of Zeng [9], the exact bound for Baskakov basis functions can be obtained as in the following lemma.

LEMMA 2.1. For all $x \in(0, \infty)$ and $k \in \mathbb{N}$,

$$
Q_{n, k}^{(\alpha)}(x) \leq \alpha p_{n, k}(x)<\frac{\alpha \sqrt{1+x}}{\sqrt{2 e} \sqrt{n x}}
$$

where the constant $1 / \sqrt{2 e}$ is the best possible.

Proof. From [9, Theorem 2], it is known that

$$
\left(\begin{array}{c}
n+k-1 \\
k
\end{array}\right) t^{k}(1-t)^{n}<\frac{1}{\sqrt{2 e}} \frac{1}{\sqrt{n t}}, \quad t \in(0,1]
$$

Replacing the variable $t$ with $x /(1+x)$ in the above inequality, we get

$$
p_{n, k}(x)<\frac{\sqrt{1+x}}{\sqrt{2 e} \sqrt{n x}}, \quad x \in(0, \infty)
$$

Also, from the fact that $\left|a^{\alpha}-b^{\alpha}\right| \leq \alpha|a-b|$ with $0 \leq a, b \leq 1, \alpha \geq 1$, it follows that

$$
Q_{n, k}^{(\alpha)}(x) \leq \alpha p_{n, k}(x)<\frac{\alpha \sqrt{1+x}}{\sqrt{2 e} \sqrt{n x}}
$$

LEMMA 2.2. For $x \in(0, \infty)$,

$$
\int_{x}^{\infty} b_{n, k}(t) d t=\sum_{j=0}^{k} p_{n, j}(x)
$$

LEMMA 2.3 [2]. The function $\mu_{n, m}(x), m \in \mathbb{N}^{0}$, can be defined as

$$
\mu_{n, m}(x)=\sum_{k=0}^{\infty} p_{n, k}(x) \int_{0}^{\infty} b_{n, k}(t)(t-x)^{m} d t .
$$


Then,

$$
\begin{gathered}
\mu_{n, 0}(x)=1, \quad \mu_{n, 1}(x)=\frac{1+x}{n-1}, \quad n>1, \\
\mu_{n, 2}(x)=\frac{2(n+1) x^{2}+2(n+2) x+2}{(n-1)(n-2)}, \quad n>2 .
\end{gathered}
$$

Consequently, for each $x \in[0, \infty), \mu_{n, m}(x)=O\left(n^{-[(m+1) / 2]}\right)$. Given any number $\lambda>2$ and $x>0$ from Lemma 2.3, in particular, for $n \geq \mathbb{N}(\lambda, x)$,

$$
\mu_{n, 2}(x) \leq \frac{\lambda x(1+x)}{n}
$$

LEMMA 2.4. Let $x \in(0, \infty)$ and $K_{n, \alpha}(x, t)=\sum_{k=0}^{\infty} Q_{n, k}^{(\alpha)}(x) b_{n, k}(t)$. Then, for $\lambda>2$ and $n>\mathbb{N}(\lambda, x)$, we have the following:

(i) $\beta_{n, \alpha}(x, y)=\int_{0}^{y} K_{n, \alpha}(x, t) d t \leq \lambda \alpha x(1+x) /\left(n(x-y)^{2}\right), 0 \leq y<x$;

(ii) $1-\beta_{n, \alpha}(x, z)=\int_{z}^{\infty} K_{n, \alpha}(x, t) d t \leq \lambda \alpha x(1+x) / n(z-y)^{2}, x \leq z<\infty$.

Proof. First, we prove (i). In view of (2.9), we have

$$
\begin{aligned}
\int_{0}^{y} K_{n, \alpha}(x, t) d t & \leq \int_{0}^{y} K_{n, \alpha}(x, t) \frac{(x-t)^{2}}{(x-y)^{2}} d t \\
& \leq \alpha(x-y)^{-2} \mu_{n, 2}(x) \\
& \leq \frac{\lambda \alpha x(1+x)}{n(x-y)^{2}}, \quad 0 \leq y<x
\end{aligned}
$$

The proof of (ii) is similar.

3. Proof of Theorem 1.1. It is easily verified [11] that

$$
\begin{aligned}
\mid B_{n, \alpha}(f, x)- & {\left[\frac{1}{\alpha+1} f(x+)+\frac{\alpha}{\alpha+1} f(x-)\right] \mid } \\
\leq & \left|B_{n, \alpha}\left(g_{x}, x\right)\right|+\frac{1}{2}|f(x+)-f(x-)| \\
& \cdot\left|B_{n, \alpha}(\operatorname{sign}(t-x), x)+\frac{\alpha-1}{\alpha+1}\right| .
\end{aligned}
$$

In order to prove the theorem, we need the estimates for $B_{n, \alpha}\left(g_{x}, x\right)$ and $B_{n, \alpha}(\operatorname{sign}(t$ $-x), x)$. We first estimate $B_{n, \alpha}(\operatorname{sign}(t-x), x)$ as follows:

$$
\begin{aligned}
B_{n, \alpha}(\operatorname{sign}(t-x), x) & =\int_{x}^{\infty} K_{n, \alpha}(x, t) d t-\int_{0}^{x} K_{n, \alpha}(x, t) d t \\
& =2 \int_{x}^{\infty} K_{n, \alpha}(x, t) d t-1, \text { because } \int_{0}^{\infty} K_{n, \alpha}(x, t) d t=1 .
\end{aligned}
$$


Using Lemma 2.2, we have

$$
\begin{aligned}
B_{n, \alpha}(\operatorname{sign}(t-x), x) & =-1+2 \sum_{k=0}^{\infty} Q_{n, k}^{(\alpha)}(x) \int_{x}^{\infty} b_{n, k}(t) d t \\
& =-1+2 \sum_{k=0}^{\infty} Q_{n, k}^{(\alpha)}(x) \sum_{j=0}^{k} p_{n, j}(x) \\
& =-1+2 \sum_{j=0}^{\infty} p_{n, j}(x) \sum_{k=j}^{\infty} Q_{n, k}^{(\alpha)}(x) \\
& =-1+2 \sum_{j=0}^{\infty} p_{n, j}(x) J_{n, j}^{\alpha}(x) .
\end{aligned}
$$

Thus,

$$
B_{n, \alpha}(\operatorname{sign}(t-x), x)+\frac{\alpha-1}{\alpha+1}=2 \sum_{j=0}^{\infty} p_{n, j}(x) J_{n, j}^{\alpha}(x)-\frac{2}{\alpha+1} \sum_{j=0}^{\infty} Q_{n, j}^{(\alpha+1)}(x)
$$

since $\sum_{k=0}^{\infty} Q_{n, k}^{(\alpha)}(x)=1$. By mean value theorem, we have

$$
Q_{n, j}^{(\alpha+1)}(x)=J_{n, j}^{\alpha+1}(x)-J_{n, j+1}^{\alpha+1}(x)=(\alpha+1) p_{n, j}(x) \gamma_{n, j}^{\alpha}(x),
$$

where $J_{n, j}^{\alpha}(x)<\gamma_{n, j}^{\alpha}(x)<J_{n, j}^{\alpha}(x)$. Therefore,

$$
\begin{aligned}
\left|B_{n, \alpha}(\operatorname{sign}(t-x), x)+\frac{\alpha-1}{\alpha+1}\right| & =2 \sum_{j=0}^{\infty} p_{n, j}(x)\left(J_{n, j}^{\alpha}(x)-\gamma_{n, j}^{\alpha}(x)\right) \\
& =2 \sum_{j=0}^{\infty} p_{n, j}(x)\left(J_{n, j}^{\alpha}(x)-J_{n, j+1}^{\alpha}(x)\right) \\
& =2 \alpha \sum_{j=0}^{\infty} p_{n, j}(x)\left(J_{n, j}(x)-J_{n, j+1}(x)\right) \\
& =2 \alpha \sum_{j=0}^{\infty} p_{n, j}^{2}(x) .
\end{aligned}
$$

Using Lemma 2.1, we have

$$
\left|B_{n, \alpha}(\operatorname{sign}(t-x), x)+\frac{\alpha-1}{\alpha+1}\right|<2 \alpha \frac{\sqrt{1+x}}{\sqrt{2 e n x}} \sum_{j=0}^{\infty} p_{n, j}(x)=\frac{\alpha \sqrt{2(1+x)}}{\sqrt{e} \sqrt{n x}} .
$$

Next, we estimate $B_{n, \alpha}\left(g_{x}, x\right)$ as follows:

$$
\begin{aligned}
B_{n, \alpha}\left(g_{x}, x\right) & =\int_{0}^{x} g_{x}(t) K_{n, \alpha}(x, t) d t \\
& =\left(\int_{0}^{x-x / \sqrt{n}}+\int_{x-x / \sqrt{n}}^{x+x / \sqrt{n}}+\int_{x+x / \sqrt{n}}^{\infty}\right) K_{n, \alpha}(x, t) g_{x}(t) d t \\
& =E_{1}+E_{2}+E_{3} .
\end{aligned}
$$


First, we estimate $E_{2}$. For $t \in[x-x / \sqrt{n}, x+x / \sqrt{n}]$, we have

$$
\left|g_{x}(t)\right| \leq V_{x-x / \sqrt{n}}^{x+x / \sqrt{n}}\left(g_{x}\right) \leq \frac{1}{n} \sum_{k=1}^{n} V_{x-x / \sqrt{k}}^{x+x / \sqrt{k}}\left(g_{x}\right)
$$

and thus

$$
\left|E_{2}\right| \leq V_{x-x / \sqrt{n}}^{x+x / \sqrt{n}}\left(g_{x}\right) \leq \frac{1}{n} \sum_{k=1}^{n} V_{x-x / \sqrt{k}}^{x+x / \sqrt{k}}\left(g_{x}\right)
$$

Next, we estimate $E_{1}$. Setting $y=x-x / \sqrt{n}$ and integrating by parts, we have

$$
E_{1}=\int_{0}^{y} g_{x}(t) d t\left(\beta_{n, \alpha}(x, t)\right)=g_{x}(y) \beta_{n, \alpha}(x, y)-\int_{0}^{y} \beta_{n, \alpha}(x, t) d t\left(g_{x}(t)\right)
$$

Since $\left|g_{x}(y)\right| \leq V_{y}^{x}\left(g_{x}\right)$, we conclude

$$
\left|E_{1}\right| \leq V_{y}^{x}\left(g_{x}\right) \beta_{n, \alpha}(x, y)+\int_{0}^{y} \beta_{n, \alpha}(x, t) d t\left(-V_{t}^{x}\left(g_{x}\right)\right)
$$

Also, $y=x-x / \sqrt{n} \leq x$, by Lemma 2.4, we get

$$
\left|E_{1}\right| \leq \frac{\alpha \lambda x(1+x)}{n(x-y)^{2}} V_{y}^{x}\left(g_{x}\right)+\frac{\alpha \lambda x(1+x)}{n} \int_{0}^{y} \frac{1}{(x-t)^{2}} d t\left(-V_{t}^{x}\left(g_{x}\right)\right) .
$$

Integrating the last integral by parts, we obtain

$$
\left|E_{1}\right| \leq \frac{\alpha \lambda x(1+x)}{n}\left(x^{-2} V_{0}^{x}\left(g_{x}\right)+2 \int_{0}^{y} \frac{V_{t}^{x}\left(g_{x}\right) d t}{(x-t)^{3}}\right) .
$$

Replacing the variable $y$ in the last integral by $x-x / \sqrt{n}$, we get

$$
\int_{0}^{x-x / \sqrt{n}} V_{t}^{x}\left(g_{x}\right)(x-t)^{-3} d t=\sum_{k=1}^{n-1} \int_{x-x / \sqrt{k}}^{x+x / \sqrt{k}} V_{x-1}^{x}\left(g_{x}\right) t^{-3} d t \leq \frac{1}{2 x^{2}} \sum_{k=1}^{n} V_{x-x / \sqrt{k}}^{x}\left(g_{x}\right) .
$$

Hence,

$$
\left|E_{1}\right| \leq \frac{2 \alpha \lambda(1+x)}{n x} \sum_{k=1}^{n} V_{x-x / \sqrt{k}}^{x}\left(g_{x}\right)
$$

Finally, we estimate $E_{3}$, and setting $z=x+x / \sqrt{n}$, we have

$$
E_{3}=\int_{z}^{\infty} g_{x}(t) K_{n, \alpha}(x, t) d t=\int_{z}^{\infty} g_{x}(t) d t\left(\beta_{n, \alpha}(x, t)\right)
$$


We define $\Delta_{n, \alpha}(x, t)$ on $[0,2 x]$ as

$$
\Delta_{n, \alpha}(x, t)= \begin{cases}1-\beta_{n, \alpha}(x, t), & 0 \leq t<2 x, \\ 0, & t=2 x\end{cases}
$$

Thus,

$$
\begin{aligned}
E_{3} & =-\int_{z}^{2 x} g_{x}(t) d t\left(\Delta_{n, \alpha}(x, t)\right)-g_{x}(2 x) \int_{2 x}^{\infty} K_{n, \alpha}(x, t) d t+\int_{2 x}^{\infty} g_{x}(t) d t\left(\beta_{n, \alpha}(x, t)\right) \\
& =E_{31}+E_{32}+E_{33} .
\end{aligned}
$$

Integrating by parts, we get

$$
E_{31}=g_{x}(z-) \Delta_{n, \alpha}(x, z-)+\int_{z}^{2 x} \hat{\Delta}_{n, \alpha}(x, t) d t\left(g_{x}(t)\right),
$$

where $\hat{\Delta}_{n, \alpha}(x, t)$ is the normalized form of $\Delta_{n, \alpha}(x, t)$. Since $\Delta_{n, \alpha}(x, z-)=\Delta_{n, \alpha}(x, z)$ and $\left|g_{x}(z-)\right| \leq V_{x}^{z-}\left(g_{x}\right)$, we obtain

$$
\left|E_{31}\right| \leq V_{x}^{z-}\left(g_{x}\right) \Delta_{n, \alpha}(x, z)+\int_{z}^{2 x} \hat{\Delta}_{n, \alpha}(x, t) d t\left(V_{x}^{t}\left(g_{x}\right)\right) .
$$

Now, using Lemma 2.4 and the fact that $\hat{\Delta}_{n, \alpha}(x, t) \leq \Delta_{n, \alpha}(x, t)$ on $[0,2 x]$, we have

$$
\begin{aligned}
\left|E_{31}\right| \leq & \frac{V_{x}^{z-}\left(g_{x}\right) \lambda x(1+x)}{n(z-x)^{2}}+\frac{\lambda \alpha x(1+x)}{n} \int_{z}^{2 x-} \frac{1}{(x-t)^{2}} d t\left(V_{x}^{t}\left(g_{x}\right)\right) \\
& +\frac{1}{2}\left[V_{x}^{2 x-}\left(g_{x}\right) \int_{2 x}^{\infty} K_{n, \alpha}(x, u) d u\right] \\
\leq & \frac{V_{x}^{z-}\left(g_{x}\right) \lambda x(1+x)}{n(z-x)^{2}}+\frac{\lambda \alpha x(1+x)}{n} \int_{z}^{2 x-} \frac{1}{(x-t)^{2}} d t\left(V_{x}^{t}\left(g_{x}\right)\right) \\
& +\frac{1}{2} V_{2 x-}^{2 x}\left(g_{x}\right) \frac{\lambda \alpha x(1+x)}{n x^{2}} \\
\leq & \frac{V_{x}^{z-}\left(g_{x}\right) \lambda x(1+x)}{n(z-x)^{2}}+\frac{\lambda \alpha x(1+x)}{n}\left[\frac{V_{x}^{2 x}\left(g_{x}\right)}{x^{2}}-\frac{V_{x}^{z-}\left(g_{x}\right)}{(z-x)^{2}}+2 \int_{z}^{2 x} \frac{V_{x}^{t}\left(g_{x}\right)}{(x-t)^{3}} d t\right] .
\end{aligned}
$$

Thus, arguing similarly as in the estimate of $E_{1}$, we get

$$
\left|E_{31}\right| \leq \frac{2 \alpha \lambda(1+x)}{n x} \sum_{k=1}^{n} V_{x}^{x+x / \sqrt{k}}\left(g_{x}\right) .
$$

Again, by Lemma 2.4, we have

$$
\left|E_{32}\right| \leq g_{x}(2 x) \frac{\alpha \lambda(1+x)}{n x} \leq \frac{\alpha \lambda(1+x)}{n x} \sum_{k=1}^{n} V_{x}^{x+x / \sqrt{k}}\left(g_{x}\right) .
$$


Finally, for $n>\beta$, we have

$$
\left|E_{33}\right| \leq M \sum_{k=1}^{\infty} Q_{n, k}^{(\alpha)}(x) \int_{2 x}^{\infty}\left[(1+t)^{\beta}+(1+x)^{\beta}\right] b_{n, k}(t) d t .
$$

Using the identity

$$
(1+t)^{\beta}-(1+x)^{\beta} \leq\left(2^{\beta}-1\right) \frac{(1+x)^{\beta}}{x^{\beta}}(t-x)^{\beta}, \text { for } 2 x \leq t,
$$

we get

$$
\begin{aligned}
\left|E_{33}\right| \leq & M \sum_{k=0}^{\infty} Q_{n, k}^{(\alpha)}(x) \int_{2 x}^{\infty}\left(2^{\beta}-1\right) \frac{(1+x)^{\beta}}{x^{\beta}}\left[(t-x)^{\beta}+2(1+x)^{\beta}\right] b_{n, k}(t) d t \\
\leq & M\left(2^{\beta}-1\right) \frac{(1+x)^{\beta}}{x^{\beta}} \sum_{k=0}^{\infty} Q_{n, k}^{(\alpha)}(x) \int_{2 x}^{\infty} b_{n, k}(t)(t-x)^{\beta} d t \\
& +2 M(1+x)^{\beta} \sum_{k=0}^{\infty} Q_{n, k}^{(\alpha)}(x) \int_{2 x}^{\infty} b_{n, k}(t) d t \\
\leq & M\left(2^{\beta}-1\right) \frac{(1+x)^{\beta}}{x^{\beta}} \sum_{k=0}^{\infty} Q_{n, k}^{(\alpha)}(x) \int_{2 x}^{\infty} b_{n, k}(t) \frac{(t-x)^{2 \beta}}{x^{\beta}} d t \\
& +2 M \frac{(1+x)^{\beta}}{x^{2}} B_{n, \alpha}\left((t-x)^{2}, x\right) \\
\leq & M\left(2^{\beta}-1\right) \frac{(1+x)^{\beta} \alpha}{x^{2 \beta}} O\left(n^{-\beta}\right)+\frac{2 M \alpha \lambda(1+x)^{\beta+1}}{n x} .
\end{aligned}
$$

Collecting the estimates of (3.1), (3.7), (3.8), (3.10), (3.16), (3.19), (3.23), (3.24), and (3.27), we get the required result.

This completes the proof of the theorem.

REMARK 3.1. It is easier to define the Bezier variants of the well-known summationintegral type operators. For example, Szász-Mirakyan-Baskakov operators $S_{n}$ and Baskakov-Szász type operators $M_{n}$ were introduced and studied in [4, 5], respectively. We may introduce their Bezier variants as follows.

(i) Szász-Mirakyan-Baskakov Bezier operators

$$
S_{n, \alpha}(f, x)=\sum_{k=0}^{\infty} R_{n, k}^{(\alpha)}(x) \int_{0}^{\infty} p_{n, k}(t) f(t) d t, \quad x \in[0, \infty),
$$

where $R_{n, k}^{(\alpha)}(x)=L_{n, k}^{\alpha}(x)-L_{n, k+1}^{\alpha}(x), L_{n, k}(x)=\sum_{j=k}^{\infty} e^{-n x}\left((n x)^{k} / k !\right)$, and $p_{n, k}(t)$ is as defined by (1.1). For further properties of $R_{n, k}^{(\alpha)}(x)$, we refer the readers to [10].

(ii) Baskakov-Szász-Bezier operators

$$
M_{n, \alpha}(f, x)=n \sum_{k=0}^{\infty} Q_{n, k}^{(\alpha)}(x) \int_{0}^{\infty} s_{n, k}(t) f(t) d t, \quad x \in[0, \infty),
$$

where $Q_{n, k}^{(\alpha)}(x)$ is defined in (1.5), and $s_{n, k}(t)=e^{-n t}\left((n t)^{k} / k !\right)$. 
But the analogous results for these operators are not possible. The main problem is in the estimation of $S_{n, \alpha}(\operatorname{sign}(t-x), x)$ and $M_{n, \alpha}(\operatorname{sign}(t-x), x)$ because we cannot relate summation of Szász (Baskakov) basis with integral of Baskakov (Szász) basis functions. That is, we cannot find result of the type [8, Lemma 5]. There may be some other techniques to solve this problem. This problem is still unresolved, and it is an open problem for the readers.

REMARK 3.2. It was observed in [2] that the operators with weight functions of Beta operators give better results in simultaneous approximation than the usual Baskakov Durrmeyer operators studied in $[7,8]$. Here, we have considered the weight functions of Beta operators, and, for $\alpha=1$, we obtain the better estimate on the rate of convergence for bounded variation functions over the main results of [8].

\section{REFERENCES}

[1] R. Bojanić and M. Vuilleumier, On the rate of convergence of Fourier-Legendre series of functions of bounded variation, J. Approx. Theory 31 (1981), no. 1, 67-79.

[2] V. Gupta, A note on modified Baskakov type operators, Approx. Theory Appl. (N.S.) 10 (1994), no. 3, 74-78.

[3] _ Rate of convergence by Baskakov-beta operators, Mathematica 37(60) (1995), no. 1-2, 109-117.

[4] V. Gupta and G. S. Srivastava, On convergence of derivative by Szász-Mirakyan Baskakov type operators, Math. Student 64 (1995), no. 1-4, 195-205.

[5] _ On simultaneous approximation by combinations of Baskakov-Szász type operators, Fasc. Math. (1997), no. 27, 29-41.

[6] A. Sahai and G. Prasad, On simultaneous approximation by modified Lupas operators, J. Approx. Theory 45 (1985), no. 2, 122-128.

[7] R. P. Sinha, P. N. Agrawal, and V. Gupta, On simultaneous approximation by modified Baskakov operators, Bull. Soc. Math. Belg. Sér. B 43 (1991), no. 2, 217-231.

[8] W. Yuankwei and G. Shunsheng, Rate of approximation of functions of bounded variation by modified Lupas operators, Bull. Austral. Math. Soc. 44 (1991), no. 2, 177-188.

[9] X.-M. Zeng, Bounds for Bernstein basis functions and Meyer-König and Zeller basis functions, J. Math. Anal. Appl. 219 (1998), no. 2, 364-376.

[10] _ On the rate of convergence of the generalized Szász type operators for functions of bounded variation, J. Math. Anal. Appl. 226 (1998), no. 2, 309-325.

[11] X.-M. Zeng and W. Chen, On the rate of convergence of the generalized Durrmeyer type operators for functions of bounded variation, J. Approx. Theory 102 (2000), no. 1, $1-12$.

[12] X.-M. Zeng and V. Gupta, Rate of convergence of Baskakov-Bezier type operators for locally bounded functions, to appear in Comput. Math. Appl.

Vijay Gupta: School of Applied Sciences, Netaji Subhas Institute of Technology, Azad Hind Fauj Marg, Sector 3 Dwarka, New Delhi 110045, India

E-mail address: vijay@nsit.ac.in 


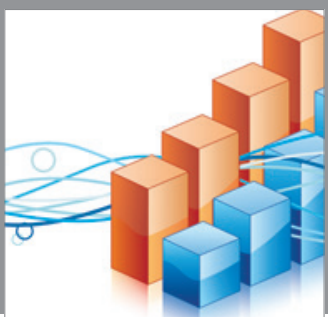

Advances in

Operations Research

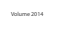

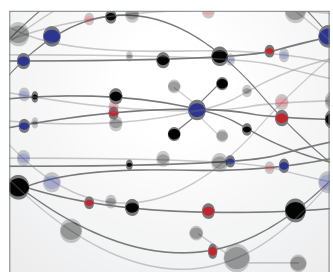

\section{The Scientific} World Journal
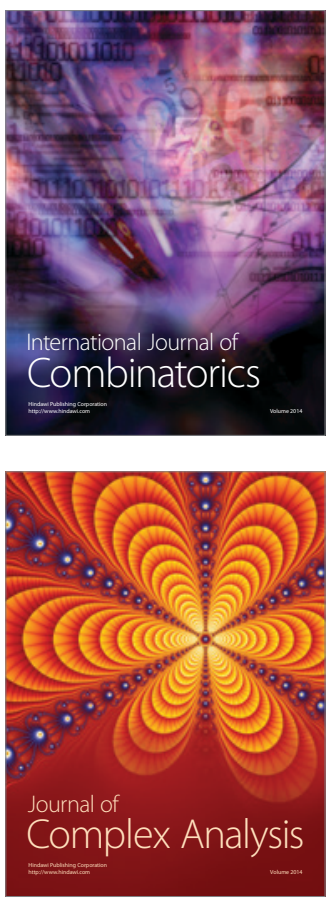

International Journal of

Mathematics and

Mathematical

Sciences
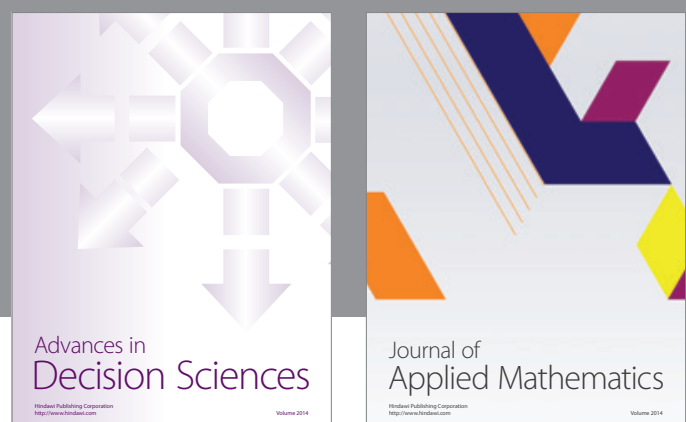

Journal of

Applied Mathematics
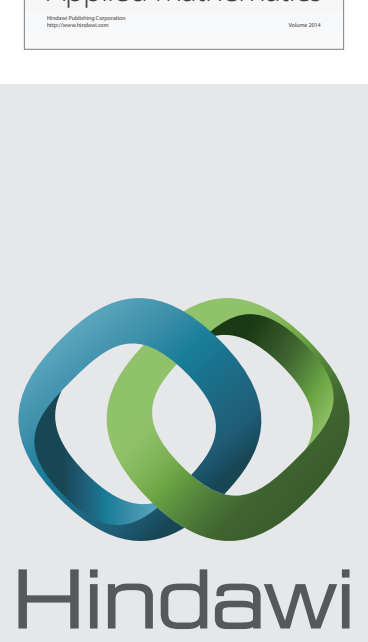

Submit your manuscripts at http://www.hindawi.com
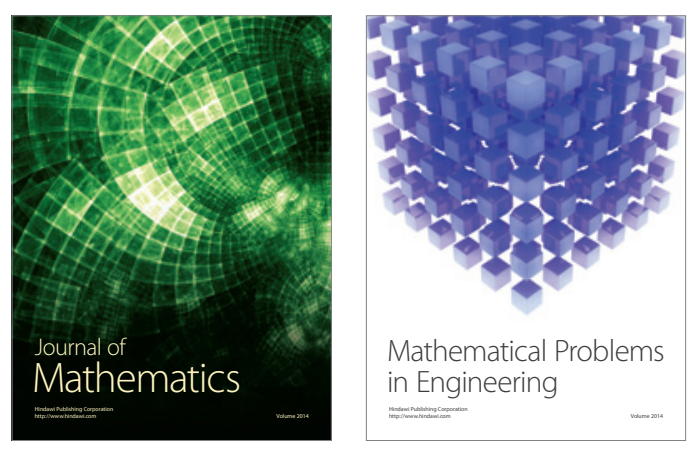

Mathematical Problems in Engineering
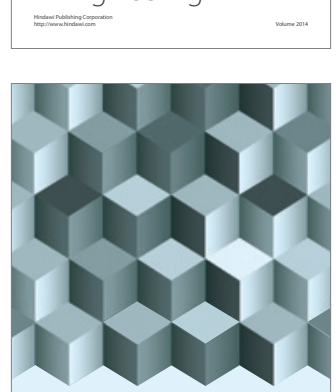

Journal of

Function Spaces
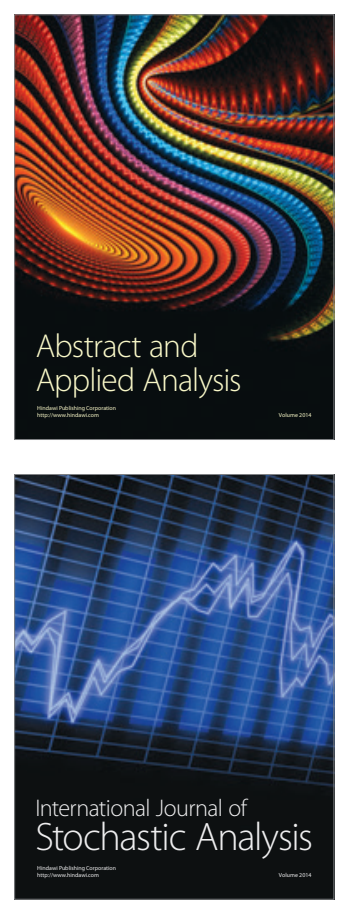

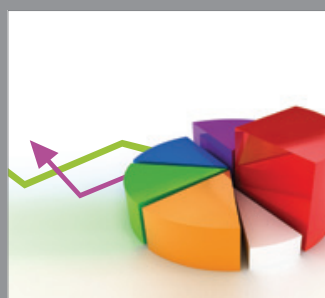

ournal of

Probability and Statistics

Promensencen
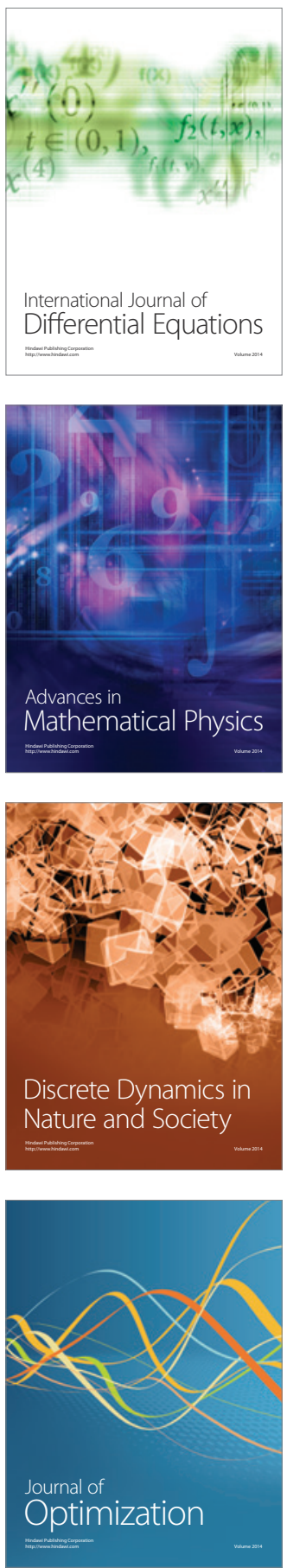